\title{
The Emission of Radiation from Diatomic Gases. III. Numerical Emissivity Calculations for Carbon Monoxide for Low Optical Densities at $300^{\circ} \mathrm{K}$ and Atmospheric Pressure*
}

\author{
S. S. Penner, M. H. Ostrander, $\dagger$ and H. S. Tsien \\ Guggenheim Jet Propulsion Center, California Instibute of Technology, Pasadena, Califormia
}

(Received September 27, 1951)

\begin{abstract}
Numerical emissivity calculations at $300^{\circ} \mathrm{K}$ and atmospheric pressure for nonoverlapping rotational lines have been carried out for CO using a dispersion formula for the line-shape representation. Use of the best available experimental data on integrated absorption and rotational line-width leads to calculated emissivities which are in excellent agreement with extrapolated empirical data published by Hottel and Ulirich. In particular, the theoretical dependence of emissivity on optical density, for small optical densities at $300^{\circ} \mathrm{K}$, has been shown to follow experimental observations with satisfactory precision.

For small optical densities the calculated emissivity is found to be proportional to the square root of the assumed rotational line-width, thus emphasizing the need for accurate line-width determinations at elevated temperatures. The limits of validity
\end{abstract}

of the treatment utilizing nonoverlapping rotational lines are defined by examining overlapping between adjacent weak and strong rotational lines.

The calculation of emissivities can be simplified by the use of approximate treatments. Thus absolute values of the emissivity can be predicted within 10 percent by utilizing a treatment for nonoverlapping, equally spaced, and equally intense lines, together with empirically determined values for the equivalent mean integrated absorption of the rotational lines of $\mathrm{CO}$. A better analytic solution, which does not involve the assumptions of equal spacing and equal intensity of the rotational lines, has been obtained by utilizing asymptotic relations for large values of modified Bessel functions.

\section{INTRODUCTION}

$\mathrm{T}$ HE general problem of emission and absorption of radiation involves two essentially distinct parts. One part of radiant heat transfer investigations is concerned with the determination of intensities if emissivities, absorptivities, and scattering coefficients are known. These studies are restricted to the problem of radiative transfer. For any defined geometric arrangement the radiative transfer problems can be formulated without difficulty. An exhaustive account of this work may be found in a recently published book by Chandrasekhar. ${ }^{1}$ The other part of the general problem is the determination of emissivities, absorptivities, and scattering coefficients, which enter into the radiative transfer calculations. Until very recently, practical applications have been based almost entirely on the use of empirically determined emissivities. These emissivities are compiled, for instance, in McAdams' well-known treatise on heat transfer. ${ }^{2}$ However, the problems involved in the theoretical calculation of emissivities were solved, in principle, a good many years ago. The results of these theoretical studies have been applied, to a limited extent, in the use of spectroscopic measurements for flame temperature determinations from line intensities ${ }^{3}$ and for the analysis of absorption and emission of radiation in the atmosphere. An important paper by

\footnotetext{
* Supported, in part, by the ONR under contract Nonr-220(O3), NR 015210 , with the California Institute of Technology.

$t$ This article uses, in part, the results of a thesis submitted by $M$. H. Ostrander in partial fulfillment of requirements for the degree of Aeronautical Engineer, California Institute of Technology, June, 1951.

'S. Chandrasekhar, Radiative Transfer (Clarendon Press, Oxford, 1950).

"H. C. Hottel, "Radiant heat transfer," in McAdams' Heat Transmission (McGraw-Hill Book Company, Inc., New York, 1942).

${ }^{3}$ For a review, containing numerous references to the original literature, see Am. J. Phys. 17, 422, 491 (1949).

W. M. Elsasser, Harvard Meteorological Studies No, 6, Blue Hill Observatory, Milton, Massachusetts, 1942.
}

Dennison, ${ }^{5}$ written more than twenty years ago, contains many of the basic theoretical relations which have been used for numerical emissivity calculations on $\mathrm{CO}$ in the present studies.

In preceding publications an approximate method for carrying out emissivity calculations at elevated total pressures, leading to complete overlapping of rotational lines, has been described. ${ }^{\text {f }} 8$ Limited comparison of calculated emissivities with experimental measurements has shown that the approximate method is, as expected, not accurately valid at atmospheric pressure for a diatomic emitter such as CO. In particular, the calculated dependence of emissivity on optical density for nonoverlapping rotational lines is not predicted correctly by a method based on the use of average absorption coefficients for entire vibration-rotation bands. ${ }^{7} \mathrm{It}$ is the purpose of the present investigation to demonstrate that the observed discrepancies between the previously proposed theory and the experiments can be removed by a more satisfactory analysis of the emission of radiation from vibration-rotation bands with rotational lines which do not overlap appreciably.

In Section II we present a summary of basic relations which are used for calculations on diatomic molecules with nonoverlapping rotational lines. Numerical emissivity calculations for $\mathrm{CO}$ at $300^{\circ} \mathrm{K}$ and a comparison with extrapolated experimental data are presented in Section III. The limits of validity of a treatment for nonoverlapping rotational lines are discussed in Section IV. In Section V approximate methods for performing emissivity calculations are considered under the conditions which are of interest for the present calculations.

${ }^{5}$ D. M. Dennison, Phys. Rev, 31, 503 (1928).

S. S. Penner, J. Appl. Phys. 21, 685 (1950); L. E. Benitez and S. S. Penner 21, 907 (1950).

'S. S. Penner, J. Appl. Mech. 18, 53 (1951).

\&. S. Penner and D. Weber, J. Appl. Phys. 22, 1164 (1951). 


\section{SUMMARY OF THEORETICAL RELATIONS}

For diatomic molecules which do not possess a $Q$ branch but a rotational line shape which may be represented by a dispersion formula, the spectral absorptions coefficient $P_{\omega}$ at the wave number $\omega$ resulting from energy transitions corresponding to the fundamental vibration-rotation band is $5,9,10$

$$
\begin{aligned}
P_{\omega}=(b / \pi) \sum_{i}\left\{S_{j}\left[\left(\omega-\omega_{j}\right)^{2}+b^{2}\right]^{-1}\right. \\
\\
\left.+S_{j-1}\left[\left(\omega-\omega_{j-1}\right)^{2}+b^{2}\right]^{-1}\right\},
\end{aligned}
$$

wheref

$$
\begin{aligned}
& S_{j}=\left(N T \pi \epsilon^{2} / 3 \mu c Q\right)\left[\left(\omega_{j} / \omega^{*}\right) j\right. \\
&\times\{\exp [-E(0, j) / k T]\}] F G\left(\omega^{*} / \omega_{e}\right), \\
& S_{j-1}=\left(N_{7} \pi \epsilon^{2} / 3 \mu c Q\right)\left[\left(\omega_{j-1} / \omega^{*}\right) j\right. \\
&\left.\times\{\exp [-E(0, j-1) / k T]\} F^{\prime} G^{\prime}\right]\left(\omega^{*} / \omega_{e}\right) .
\end{aligned}
$$

Here $N_{T}=$ total number of molecules per unit volume per unit of total pressure; $\mu=$ reduced mass of the diatomic molecule under discussion; $c=$ velocity of light; $\epsilon=$ rate of change of electric moment with internuclear distance; $b=$ rotational half-width in $\mathrm{cm}^{-1} ; \omega_{e}=$ wave number corresponding to an infinitesimal oscillation at the equilibrium interatomic distance; $\omega^{*}=$ wave number of the (forbidden) transition $j=0 \rightarrow j=0$ and $n=0 \longrightarrow n=1$, where $j$ and $n$ denote, respectively, rotational and vibrational quantum numbers; $\omega_{j}=$ wave number corresponding to the transitions $j \rightarrow j-1$, $n=0 \rightarrow n=1 ; \omega_{j-1}=$ wave number corresponding to the transitions $j-1 \rightarrow j, n=0 \rightarrow n=1 ; E(n, j)=$ energy of the $n$th vibrational and $j$ th rotational level;

$$
\begin{aligned}
F & =1+8 \gamma j[1+(5 \gamma j / 4)-(3 \gamma / 4)] ; \\
F^{\prime} & =1-8 \gamma j[1-(5 \gamma j / 4)-(3 \gamma / 4)] ;
\end{aligned}
$$

$\gamma=h / 8 \pi^{2} I c \omega_{e}$ where $I$ represents the equilibrium moment of inertia of the radiating molecule;

$$
\begin{aligned}
G & =1-\exp \left(-h c \omega_{j} / k T\right) ; \\
G^{\prime} & =1-\exp \left(-h c \omega_{j-1} / k T ;\right.
\end{aligned}
$$

$Q=$ complete internal partition function referred to zero as reference state.

At elevated temperatures the actual value of $P_{\omega}$ must be obtained by adding a small number of series of the type given in Eq. (1) corresponding to vibrational transitions of the form $n \rightarrow n+1$. Furthermore, contributions from vibration-rotation bands for which $n \rightarrow n+2, n \rightarrow n+3$, etc., become important for large optical densities. Detailed applications are discussed in reference 9.

The total equilibrium intensity of radiation emitted per unit surface area per unit time into a solid angle of

' S. S. Penner, J. Chem. Phys. 19, 272, 1434 (1951).

${ }_{10} \mathrm{~J}$. R. Oppenheimer, Proc. Cambridge Phil. Soc. 23, 327 (1926).

$\ddagger$ Equations (2a) and (2b) differ from the corresponding relations obtained from the treatment of B. L. Crawford, Jr., and H. L. Dinsmore (J. Chem. Phys. 18, 983, 1682 (1950)) through the occurrence of the factors $F$ and $F^{\prime}$, respectively. These factors are nearly equal to unity for the values of $j$ which are important for the present studies.
$2 \pi$ steradians in the wave number interval between $\omega$ and $\omega+d \omega$ by heated gases distributed uniformly and of optical density $X$ ( $X=$ product of partial pressure $p$ of emitter and radiation path length $L$ ) is given by the well-known relation

$$
R(\omega)=R^{0}(\omega)\left[1-\exp \left(-P_{\omega} X\right)\right] .
$$

Here $R^{0}(\omega)$ is the intensity of radiation emitted by a blackbody in the wave number interval between $\omega$ and $\omega+d \omega$ into a solid angle of $2 \pi$ steradians per unit surface area per unit time. It is given in analytic form by the Planck blackbody distribution function. The engineering emissivity $E$ is defined by the relation

$$
E=\int_{0}^{\infty} R(\omega) d \omega / \sigma T^{4}
$$

where $\sigma$ is the Stefan-Boltzmann constant.

Subject to the approximations and assumptions involved in the use of Eq. (1), it is evident that the problem of emissivity calculations reduces to the evaluation of integrals of the type appearing in Eq. (4). This integration can be accomplished if the width of the rotational lines is so small that they may be treated as being completely separated. The results obtained for nonoverlapping rotational lines are useful at low pressures. ${ }^{4.9,11}$ The integrals can also be evaluated, approximately, if the pressures are so high that very extensive overlapping of the rotational lines occurs. The results obtained in this case are useful for emissivity calculations at elevated pressures in rocket combustion chambers. ${ }^{6-8}$ Emissivity calculations at intermediate pressures are more difficult to carry out.

We proceed by considering the problem of emissivity calculations for nonoverlapping rotational lines. In order to evaluate the infinite integral

$$
\int_{0}^{\infty} R(\omega) d \omega=\int_{0}^{\infty} R^{0}(\omega)\left[1-\exp \left(-P_{\omega} X\right)\right] d \omega,
$$

for nonoverlapping rotational lines, the approximation is first made that $R^{0}(\omega)$ may be treated as constant in the wave number interval for which $\exp \left(-P_{\omega} X\right)$ is sensibly different from unity. Replacing $R^{0}(\omega)$ by an appropriate average value introduces negligibly small errors into the emissivity calculations for nonoverlapping rotational lines at low pressures, since the intervals $\Delta \omega$ for which $P_{\omega} X$ is significantly different from zero are exceedingly small. For example, at atmospheric pressure and room temperature $\Delta \omega$ is of the order of $0.3 \mathrm{~cm}^{-1}$ for $\mathrm{CO}$. The change of $R^{0}(\omega)$ in wave number intervals of this size is less than 1 percent.

At low pressures the rotational lines are so narrow that the only term contributing to $R^{0}(\omega)$, for example, in the vicinity of the rotational line centered at $\omega_{j}$ is the term $S_{j}\left[\left(\omega-\omega_{j}\right)^{2}+b^{2}\right]^{-1}$. Hence we can break up the integration interval in such a way that each subinterval

11 R. Ladenburg and F. Reiche, Ann. Physik 42, 181 (1913). 
TABLE I. Integrated absorption for the rotational lines of the fundamental of $\mathrm{CO}$ at $300^{\circ} \mathrm{K}$.

\begin{tabular}{cccccc}
\hline $\begin{array}{c}\text { Transi- } \\
\text { tion } \\
j \rightarrow j-1\end{array}$ & $\begin{array}{c}10^{3} A_{i} \\
\left(\mathrm{~cm}^{-2}\right. \\
\left.\text { atmos }^{-1}\right)\end{array}$ & $\begin{array}{c}S_{j} \\
\left(\mathrm{~cm}^{-2}\right. \\
\left.\text { atmos }^{-1}\right)\end{array}$ & $\begin{array}{c}\text { Transition } \\
j-1 \rightarrow j\end{array}$ & $\begin{array}{c}1^{3} A_{i-1} \\
\left(\mathrm{~cm}^{-2}\right. \\
\left.\text { atmos }^{-1}\right)\end{array}$ & $\begin{array}{c}S_{j-1} \\
\left(\mathrm{~cm}^{-2}\right. \\
\left.\text { atmos }^{-1}\right)\end{array}$ \\
\hline $\begin{array}{c}1 \rightarrow 0 \\
2 \rightarrow 1\end{array}$ & 5.528 & 2.064 & $0 \rightarrow 1$ & 5.572 & 2.081 \\
$3 \rightarrow 2$ & 10.71 & 4.000 & $1 \rightarrow 2$ & 10.88 & 4.062 \\
$4 \rightarrow 3$ & 15.28 & 5.705 & $2 \rightarrow 3$ & 15.64 & 5.841 \\
$5 \rightarrow 4$ & 21.79 & 7.101 & $3 \rightarrow 4$ & 19.62 & 7.326 \\
$6 \rightarrow 5$ & 23.51 & 8.135 & $4 \rightarrow 5$ & 22.65 & 8.457 \\
$7 \rightarrow 6$ & 24.24 & 9.050 & $5 \rightarrow 6$ & 24.64 & 9.200 \\
$8 \rightarrow 7$ & 24.00 & 8.960 & $7 \rightarrow 7$ & 25.58 & 9.551 \\
$9 \rightarrow 8$ & 22.97 & 8.577 & $8 \rightarrow 9$ & 25.54 & 9.535 \\
$10 \rightarrow 9$ & 21.32 & 7.960 & $9 \rightarrow 10$ & 24.63 & 9.199 \\
$11 \rightarrow 10$ & 19.23 & 7.180 & $10 \rightarrow 11$ & 20.94 & 8.603 \\
$12 \rightarrow 11$ & 16.88 & 6.310 & $11 \rightarrow 12$ & 18.53 & 6.919 \\
$13 \rightarrow 12$ & 14.45 & 5.397 & $12 \rightarrow 13$ & 15.99 & 5.969 \\
$14 \rightarrow 13$ & 12.07 & 4.509 & $13 \rightarrow 14$ & 13.46 & 5.025 \\
$15 \rightarrow 14$ & 9.851 & 3.678 & $14 \rightarrow 15$ & 11.06 & 4.131 \\
$16 \rightarrow 15$ & 7.857 & 2.934 & $15 \rightarrow 16$ & 8.889 & 3.319 \\
$17 \rightarrow 16$ & 6.127 & 2.288 & $16 \rightarrow 17$ & 6.986 & 2.608 \\
$18 \rightarrow 17$ & 4.673 & 1.745 & $17 \rightarrow 18$ & 5.370 & 2.005 \\
$19 \rightarrow 18$ & 3.490 & 1.303 & $18 \rightarrow 19$ & 4.039 & 1.508 \\
$20 \rightarrow 19$ & 2.551 & 0.9525 & $19 \rightarrow 20$ & 2.975 & 1.111 \\
$22 \rightarrow 21$ & 1.281 & 0.4783 & $21 \rightarrow 22$ & 1.622 & 0.6057 \\
$24 \rightarrow 23$ & 0.5924 & 0.2212 & $23 \rightarrow 24$ & 0.773 & 0.2885 \\
$26 \rightarrow 25$ & 0.2529 & 0.0944 & $25 \rightarrow 26$ & 0.340 & 0.1269 \\
$28 \rightarrow 27$ & 0.0998 & 0.0373 & $27 \rightarrow 28$ & 0.138 & 0.0516 \\
$30 \rightarrow 29$ & 0.0364 & 0.0136 & $29 \rightarrow 30$ & 0.052 & 0.0195 \\
$35 \rightarrow 34$ & 0.0021 & 0.0078 & $34 \rightarrow 35$ & 0.001 & 0.0052 \\
$40 \rightarrow 39$ & 0.0001 & 0.0037 & $39 \rightarrow 40$ & 0.000 & 0.0000 \\
& & & & & \\
\hline
\end{tabular}

is centered about one of the wave numbers $\omega_{j}, \omega_{j-1} .{ }^{9}$ Thus the following close approximation is obtained

$$
\begin{aligned}
\int_{0}^{\infty} R(\omega) d \omega & =\sum_{\Delta \omega_{i}}\left\{R^{0}\left(\omega_{j}\right) \int_{\Delta \omega_{i}}\left[1-\exp \left(-P_{j} X\right)\right] d \omega\right. \\
& \left.+R^{0}\left(\omega_{j-1}\right) \int_{\Delta \omega_{i}}\left[1-\exp \left(-P_{j-1} X\right)\right] d \omega\right\},
\end{aligned}
$$

where $R^{0}\left(\omega_{j}\right)$ and $R^{0}\left(\omega_{j-1}\right)$ denote, respectively, the spectral intensities of a blackbody radiator evaluated at the line centers corresponding to the indicated transitions. Similarly, $P_{j}$ and $P_{j-1}$ are the characteristic values of the spectral absorption coefficients valid if only the indicated transitions occurred. The subintervals $\Delta \omega_{i}$ are chosen conveniently to extend from a wave number midway between two line centers to the adjacent wave numbers which are located similarly. For nonoverlapping lines the error involved in replacing

$$
\int_{\Delta \omega_{i}}\left[1-\exp \left(-P_{j} X\right)\right] d \omega \text { by } \int_{-\infty}^{\infty}\left[1-\exp \left(-P_{j} X\right)\right] d \omega,
$$

etc., is negligibly small. The magnitude of this error increases with increasing optical density $X$ and can be estimated readily by numerical calculations. Equation (6) now becomes

$$
\begin{aligned}
\int_{0}^{\infty} R(\omega) d \omega & =\sum_{j}\left\{R^{0}\left(\omega_{j}\right) \int_{-\infty}^{\infty}\left[1-\exp \left(-P_{j} X\right)\right] d \omega\right. \\
& \left.+R^{0}\left(\omega_{j-1}\right) \int_{-\infty}^{\infty}\left[1-\exp \left(-P_{j-1} X\right)\right] d \omega\right\}
\end{aligned}
$$

For fixed values of $b$ the infinite integrals appearing in Eq. (7) are readily evaluated with the result $t^{4,9,11}$

$$
\begin{array}{r}
\int_{0}^{\infty} R(\omega) d \omega=2 \pi b \sum_{j=1}^{\infty}\left\{R^{0}\left(\omega_{j}\right) x_{j}\right. \\
\times \exp \left(-x_{j}\right)\left[I_{0}\left(x_{j}\right)+I_{1}\left(x_{j}\right)\right]+R^{0}\left(\omega_{j-1}\right) x_{j-1} \\
\left.\quad \times \exp \left(-x_{j-1}\right)\left[I_{0}\left(x_{j-1}\right)+I_{1}\left(x_{j-1}\right)\right]\right\},
\end{array}
$$

where

$$
x_{j}=S_{j} X / 2 \pi b, \quad x_{j-1}=S_{j-1} X / 2 \pi b,
$$

and $I_{0}, I_{1}$ denote modified Bessel functions of the first kind. Calculations of the emissivity $E$ from Eqs. (4) and (8), which neglect contributions from upper harmonics, can now be carried out by utilizing intensity ${ }^{12}$ and line-width data ${ }^{13}$ obtained at room temperature. The contribution to the emissivity $E$ from the first overtone is obtained by adding to Eq. (8) a series which is of the same form as Eq. (8), except that the integrated absorption $S_{j}$ is replaced by $S_{j}{ }^{\prime}$ and $S_{j-1}$ by $S_{j-1}{ }^{\prime}$ where

\begin{tabular}{|c|c|c|c|}
\hline$\underset{j \rightarrow j-1}{\text { Transition }}$ & $\begin{array}{c}10^{2} S_{i} \\
\left(\mathrm{~cm}^{-2} \text { atmos }^{-1}\right)\end{array}$ & $\begin{array}{c}\text { Transition } \\
j-1 \rightarrow j\end{array}$ & $\left(\mathrm{~cm}^{10^{2} S_{j-1}}\right.$-atmos-1) \\
\hline $1 \rightarrow 0$ & 1.428 & $0 \rightarrow 1$ & 1.440 \\
\hline $2 \rightarrow 1$ & 2.768 & $1 \rightarrow 2$ & 2.811 \\
\hline $3 \rightarrow 2$ & 3.948 & $2 \rightarrow 3$ & 4.042 \\
\hline $4 \rightarrow 3$ & 4.914 & $3 \rightarrow 4$ & 5.070 \\
\hline $5 \rightarrow 4$ & 5.629 & $4 \rightarrow 5$ & 5.852 \\
\hline $6 \rightarrow 5$ & 6.076 & $5 \rightarrow 6$ & 6.367 \\
\hline $7 \rightarrow 6$ & 6.263 & $6 \rightarrow 7$ & 6.610 \\
\hline $8 \rightarrow 7$ & 6.201 & $7 \rightarrow 8$ & 6.598 \\
\hline $9 \rightarrow 8$ & 5.935 & $8 \rightarrow 9$ & 6.365 \\
\hline $10 \rightarrow 9$ & 5.508 & $9 \rightarrow 10$ & 5.953 \\
\hline $11 \rightarrow 10$ & 4.968 & $10 \rightarrow 11$ & 5.411 \\
\hline $12 \rightarrow 11$ & 4.366 & $11 \rightarrow 12$ & 4.788 \\
\hline $13 \rightarrow 12$ & 3.735 & $12 \rightarrow 13$ & 4.130 \\
\hline $14 \rightarrow 13$ & 3.120 & $13 \rightarrow 14$ & 3.477 \\
\hline $15 \rightarrow 14$ & 2.545 & $14 \rightarrow 15$ & 2.859 \\
\hline $16 \rightarrow 15$ & 2.030 & $15 \rightarrow 16$ & 2.297 \\
\hline $17 \rightarrow 16$ & 1.583 & $16 \rightarrow 17$ & 1.805 \\
\hline $18 \rightarrow 17$ & 1.208 & $17 \rightarrow 18$ & 1.388 \\
\hline $19 \rightarrow 18$ & 0.9017 & $18 \rightarrow 19$ & 1.0437 \\
\hline $20 \rightarrow 19$ & 0.6591 & $19 \rightarrow 20$ & 0.7688 \\
\hline $22 \rightarrow 21$ & 0.3310 & $21 \longrightarrow 22$ & 0.4191 \\
\hline $24 \rightarrow 23$ & 0.1531 & $23 \rightarrow 24$ & 0.1996 \\
\hline $26 \rightarrow 25$ & 0.0653 & $25 \rightarrow 26$ & 0.0878 \\
\hline $28 \rightarrow 27$ & 0.0258 & $27 \rightarrow 28$ & 0.0357 \\
\hline $30 \rightarrow 29$ & 0.0094 & $29 \rightarrow 30$ & 0.0135 \\
\hline $35 \rightarrow 34$ & 0.0054 & $34 \rightarrow 35$ & 0.0036 \\
\hline $40 \rightarrow 39$ & 0.0028 & $39 \longrightarrow 40$ & 0.0000 \\
\hline
\end{tabular}
the primed quantities identify appropriate values for the vibrational transition $0 \longrightarrow 2$.

\section{REPRESENTATIVE EMISSIVITY CALCULATIONS FOR CO AT $300^{\circ} \mathrm{K}$}

For the present calculations the spectroscopic constants of Sponer were used. ${ }^{14}$ Since these data have been

TABLE II. Integrated absorption for the rotational lines of the first overtone of $\mathrm{CO}$ at $300^{\circ} \mathrm{K}$.

${ }^{12}$ S. S. Penner and D. Weber, J. Chem. Phys. 19, 807, 817, 974 (1951).

${ }^{13}$ S. S. Penner and D. Weber, J. Chem. Phys. 19, 1351, 1361 (1951)

14 H. Sponer, Molekülspektren (Verlag Julius Springer, Berlin, 
revised recently, ${ }^{15,16}$ all of the wave numbers of line centers, as well as the values of blackbody radiation from the line centers, will be somewhat in error. However, the over-all effect on the calculated values of emissivity is certainly negligibly small (less than 0.1 percent).

Assuming the validity of the ideal gas law and using recently published integrated intensity data ${ }^{12}$ for the fundamental vibration-rotation band of $\mathrm{CO}$, it is found that

$$
\left(\omega^{*} / \omega_{e}\right)\left(N_{T} \pi \epsilon^{2} / 3 \mu c^{2} Q\right)=371.9 \mathrm{~cm}^{-2} \text { atmos }^{-1} .
$$

The terms appearing in the large square brackets of Eqs. (2a) and (2b) are designated as $A_{j}$ and $A_{j-1}$, respectively. Numerical values of $A_{j}, A_{j-1}$, and of $S_{j}, S_{j-1}$ at $300^{\circ} \mathrm{K}$ for the fundamental vibration-rotation band of CO are listed in Table I.

The integrated absorption for the rotational lines of the first overtone, $S_{j}{ }^{\prime}$ and $S_{j-1}{ }^{\prime}$, are obtained, in close approximation, from $S_{j}$ and $S_{j-1}$, respectively, by multiplying by the ratio of integrated absorption of the first overtone to the integrated absorption for the fundamental. The experimentally observed ratio ${ }^{12}$ is $1.64 / 237$ $=6.92 \times 10^{-3}$. Using this ratio, the results summarized in Table II are obtained for $S_{j}^{\prime}$ and $S_{j-1}{ }^{\prime}$ at $300^{\circ} \mathrm{K}$.

By utilizing the intensity data listed in Tables I and II, the emissivity has been calculated ${ }^{17}$ from Eqs. (4) and (8) as a function of optical density and rota-

TABLE III. Calculated and extrapolated emissivities for $\mathrm{CO}$ at $300^{\circ} \mathrm{K}$ as a function of optical density and rotational halfwidth.

\begin{tabular}{|c|c|c|}
\hline $\begin{aligned} T & =300^{\circ} \mathrm{K} \\
b & =0.06 \mathrm{~cm}^{-1} \\
X & =p L(\mathrm{~cm}-\mathrm{atmos})\end{aligned}$ & $\begin{array}{c}E \\
\text { (Calculated) }\end{array}$ & $\begin{array}{c}E \\
\text { (Extrapolated from } \\
\text { reference 18) }\end{array}$ \\
\hline $\begin{array}{l}0.1 \\
0.5 \\
2.0 \\
6.0\end{array}$ & $\begin{array}{l}3.62 \times 10^{-4} \\
9.30 \times 10^{-4} \\
1.92 \times 10^{-3} \\
3.43 \times 10^{-3}\end{array}$ & $\begin{array}{l}4.1 \times 10^{-4} \\
1.05 \times 10^{-3} \\
2.2 \times 10^{-3} \\
3.35 \times 10^{-3}\end{array}$ \\
\hline $\begin{array}{c}T=300^{\circ} \mathrm{K} \\
b=0.07 \mathrm{~cm}^{-1} \\
X=p l\left(\mathrm{~cm}^{-a t m o s}\right) \\
0.1 \\
0.5 \\
2.0 \\
6.0 \\
30.0 \\
70.0\end{array}$ & $\begin{array}{l}3.81 \times 10^{-4} \\
9.90 \times 10^{-4} \\
2.03 \times 10^{-3} \\
3.64 \times 10^{-3} \\
8.24 \times 10^{-3} \\
1.26 \times 10^{-2}\end{array}$ & $\begin{array}{l}4.1 \times 10^{-4} \\
1.05 \times 10^{-3} \\
2.20 \times 10^{-3} \\
3.35 \times 10^{-3} \\
5.8 \times 10^{-3} \\
7.9 \times 10^{-3}\end{array}$ \\
\hline $\begin{array}{c}T=300^{\circ} \mathrm{K} \\
b=0.08 \mathrm{~cm}^{-1} \\
X=p L\left(\mathrm{~cm}^{\text {-atmos})}\right. \\
0.1 \\
0.5 \\
2.0 \\
6.0 \\
30.0 \\
70.0\end{array}$ & $\begin{array}{l}4.13 \times 10^{-4} \\
1.08 \times 10^{-3} \\
2.21 \times 10^{-3} \\
3.90 \times 10^{-3} \\
8.84 \times 10^{-3} \\
1.35 \times 10^{-2}\end{array}$ & $\begin{array}{l}4.1 \times 10^{-4} \\
1.05 \times 10^{-3} \\
2.2 \times 10^{-3} \\
3.35 \times 10^{-3} \\
5.9 \times 10^{-3} \\
7.9 \times 10^{-3}\end{array}$ \\
\hline
\end{tabular}

${ }^{15}$ K. N. Rao, J. Chem. Phys. 18, 213 (1950).

${ }^{10}$ Silverman, Plyler, and Benedict, Paper presented before the Symposium on Molecular Structure and Spectroscopy, Columbus, Ohio, 1951.

${ }_{17}$ Further details concerning this calculation may be found in the thesis of M. H. Ostrander.

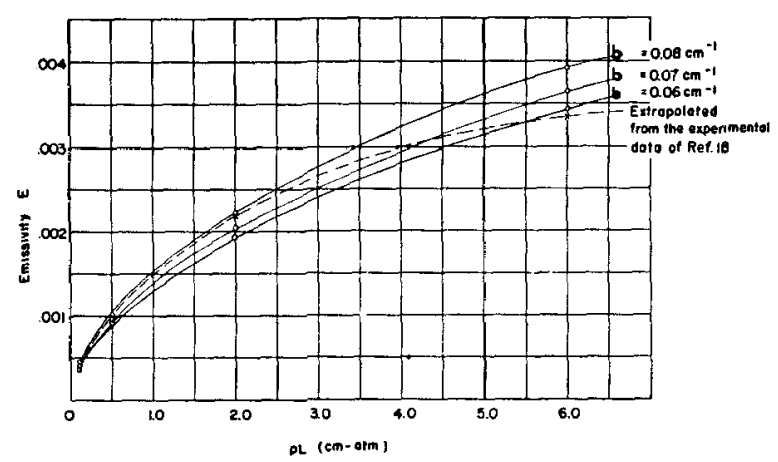

Fig. 1. Emissivity as a function of optical density at $300^{\circ} \mathrm{K}$ and atmospheric pressure.

tional half-width. The results of these calculations are summarized in Table III and plotted in Fig. 1, where they are compared with extrapolated empirical data reported by Ullrich. ${ }^{18}$

Reference to the data listed in Table III and shown in Fig. 1 indicates that the calculated emissivity is proportional to the square root of the assumed value of the rotational half-width. Almost exact agreement between calculated and observed emissivities, for small optical densities, is obtained for $b=0.076 \mathrm{~cm}^{-1}$ at atmospheric pressure. This value is in fair agreement with infrared line-width measurements on $\mathrm{CO}$ obtained by standard techniques. ${ }^{13,19}$ We therefore arrive at the important conclusion that emissivities calculated from spectroscopic data are in quantitative agreement with empirically determined results, well within the claimed limits of accuracy of the latter measurements. The fact that the calculated emissivities appear to be too large for optical densities in excess of $2 \mathrm{~cm}$-atmos is the result of failure of the assumption that no overlapping occurs between rotational lines. This matter will be discussed in Section IV.

Reference to the data listed in Table III and plotted in Fig. 1 shows that the emissivity $E$ is very accurately a linear function of $(X)^{\frac{1}{2}}$. This result, together with the observation that $E$ is proportional to the square root of the rotational half-width, is obviously in accord with Elsasser's well-known square-root law for nonoverlapping, equally intense, and equally spaced rotational lines. ${ }^{4}$ Unfortunately, the simplified treatment ${ }^{4}$ cannot be used to predict quantitatively (cf. Section VA for details) the slope of a plot of $E$ vs $(X)^{\frac{1}{2}}$ in terms of the known basic spectroscopic constants. $\$$

The results of the present calculations are of considerable practical importance. They clearly show that, for small optical densities, the emissivity is a sensitive function of rotational half-width. Thus we must conclude that not only is it not justified to extrapolate

${ }^{18}$ W. Ulirich, Dr. Sci. thesis, M.I.T., Cambridge, Massachusetts, 1935.

${ }^{19}$ L. A. Matheson, Phys. Rev. 40, 813 (1932).

$\S$ The same difficulty arises also if attempts are made to calculate the rotational half-width from experimental measurements of transmission. Compare reference 13. 


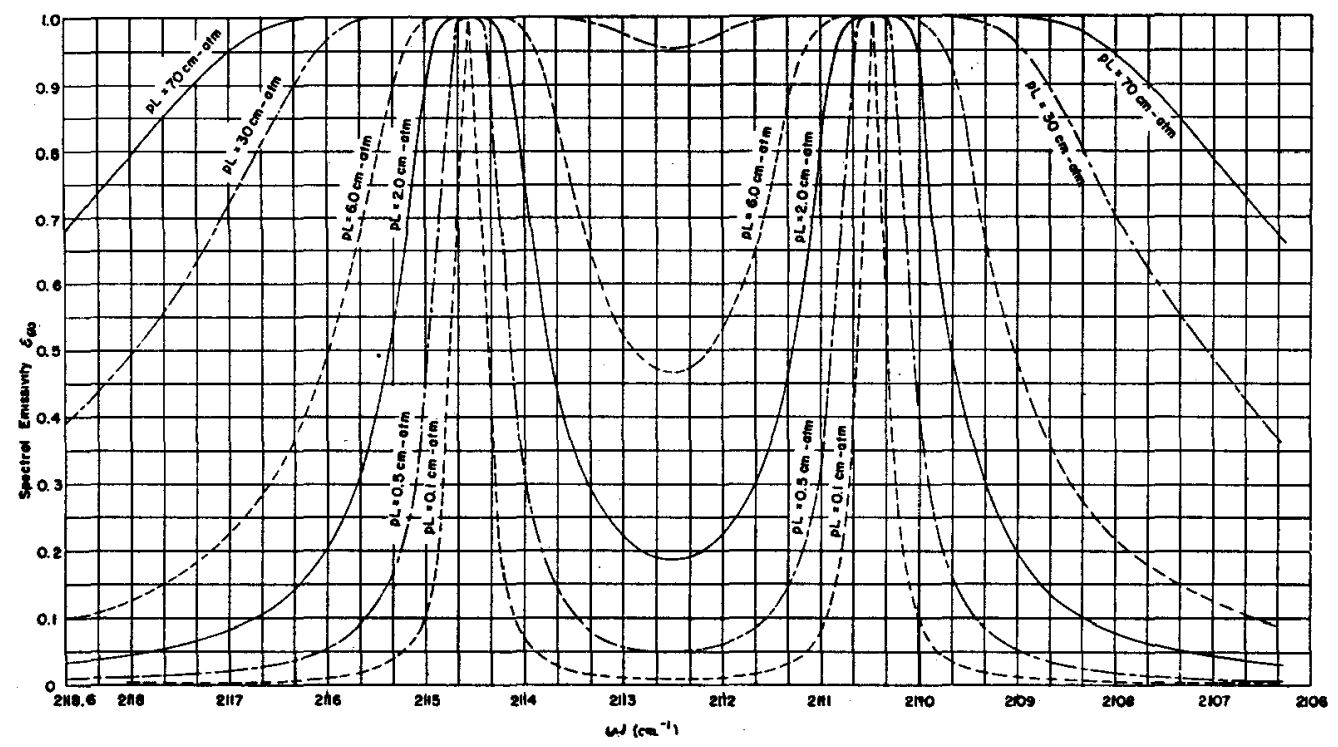

FIc. 2. Spectral emissivity $\epsilon_{\omega}$ as a function of $\omega$ and optical density for two adjacent intense rotational lines $(n=$ $0 \rightarrow n=1, j=8 \rightarrow j=7$ and $j=7 \rightarrow j=6$ ) at $300^{\circ} \mathrm{K}$ and atmospheric pressure. emissivities indiscriminately over temperature, pressure, or optical density intervals by using unsound simplified equations, but it is also essential to consider the problem with proper regard for the concentration of infrared-inert gases.

\section{LIMITS OF VALIDITY OF THE TREATMENT FOR NONOVERLAPPING ROTATIONAL LINES FOR CO ${ }^{17}$}

To illustrate the effect of overlapping on $P_{\omega}$, representative calculations involving the contributions to $P_{\omega}$ from two adjacent rotational lines have been carried out. In this case the correct expression for the spectral emissivity is evidently

$$
\epsilon_{\omega}=1-\exp \left[-\left(P_{\omega_{1}}+P_{\omega_{2}}\right)\right] X,
$$

where $P_{\omega_{1}}$ and $P_{\omega_{2}}$ represent, respectively, the values of the spectral absorption coefficients arising from each of the two rotational lines separately. If the two rotational lines are separated sufficiently to permit neglect of overlapping, then the spectral emissivity is represented by the relation

$$
\epsilon_{\omega}{ }^{\prime}=1-\exp \left(-P_{\omega_{1} X} X\right)+1-\exp \left(-P_{\omega_{2}} X\right) .
$$

By evaluating the differences $\epsilon_{\omega}^{\prime}-\epsilon_{\omega}$ from Eqs. (9) and (10) we can determine the error incurred in making numerical emissivity calculations, if overlapping between two adjacent rotational lines is ignored. The resulting expression,

$\epsilon_{\omega}{ }^{\prime}-\epsilon_{\omega}=\left(P_{\omega_{1}} P_{\omega_{2}}\right) X^{2}$

$$
-\left(P_{\omega_{1}}^{2} P_{\omega_{2}}+P_{\omega_{1}} P_{\omega_{2}}^{2}\right) X^{3} / 2+\cdots,
$$

is useful for small values of $P_{\omega_{1}} X$ and $P_{\omega_{2}} X$.

Emissivity calculations for two rotational lines based on the correct expression given in Eq. (9) can be used as a qualitative guide in ascertaining the limits of validity of theoretical calculations based on a treatment for nonoverlapping rotational lines. Representative values of $\epsilon_{\omega}$ calculated from Eq. (9) are shown in Figs. 2 and 3 as a function of $\omega$ with $X$ treated as a variable parameter. Reference to Fig. 2 indicates that the emissivity at a point midway between the rotational line centers which are characterized by the transitions $n=0 \rightarrow n=1, j=8 \rightarrow j=7$, and $j=7 \rightarrow j=6$, respectively, remains very small (i.e., $\epsilon_{\omega}<0.2$ ) for $X=2 \mathrm{~cm}$-atmos. Since the indicated transitions correspond to the two most intense rotational lines, it appears justifiable to conclude that emissivity calculations based on the use of Eq. (8) will yield valid results at least for $X \leq 2 \mathrm{~cm}$ atmos. Calculations similar to those shown in Fig. 2 for intense adjacent rotational lines are shown in Fig. 3 for weak rotational lines, namely, for the lines corresponding to the transitions $n=0 \rightarrow n=1, j=19 \rightarrow j=18$, and $j=18 \rightarrow j=17$. As is to be expected, the emissivity $\epsilon_{\omega}$ is represented more adequately by Eq. (10) for larger values of $X$ than it was for the more intense rotational lines described in Fig. 2.

Although the precise evaluation of errors arising from the use of Eq. (8) is difficult to carry out, it is evident from the data described in Figs. 2 and 3 that the calculated values of the emissivity are reliable at least to $X=2 \mathrm{~cm}$-atmos and probably to somewhat larger values. This conclusion is borne out by the comparison between calculated and observed emissivities described in Section III.

\section{APPROXIMATE EMISSIVITY CALCULATIONS FOR DIATOMIC MOLECULES WITH NONOVERLAPPING ROTATIONAL LINES}

The numerical work involved in the emissivity calculations indicated in Section III is very heavy. It is thus worthwhile to consider the possibility of developing approximate analytical expressions which avoid much of the tedious computations. In this section we shall present two such approximations, in the order of increasing accuracy. 
Fig. 3. Spectral emissivity $\epsilon_{\omega}$ as a function of $\omega$ and optical density for two adjacent weak rotational lines $(n=0$ $\rightarrow n=1, j=19 \rightarrow j=18$ and $j=18 \rightarrow j=17$ ) at $300^{\circ} \mathrm{K}$ and atmospheric pressure.

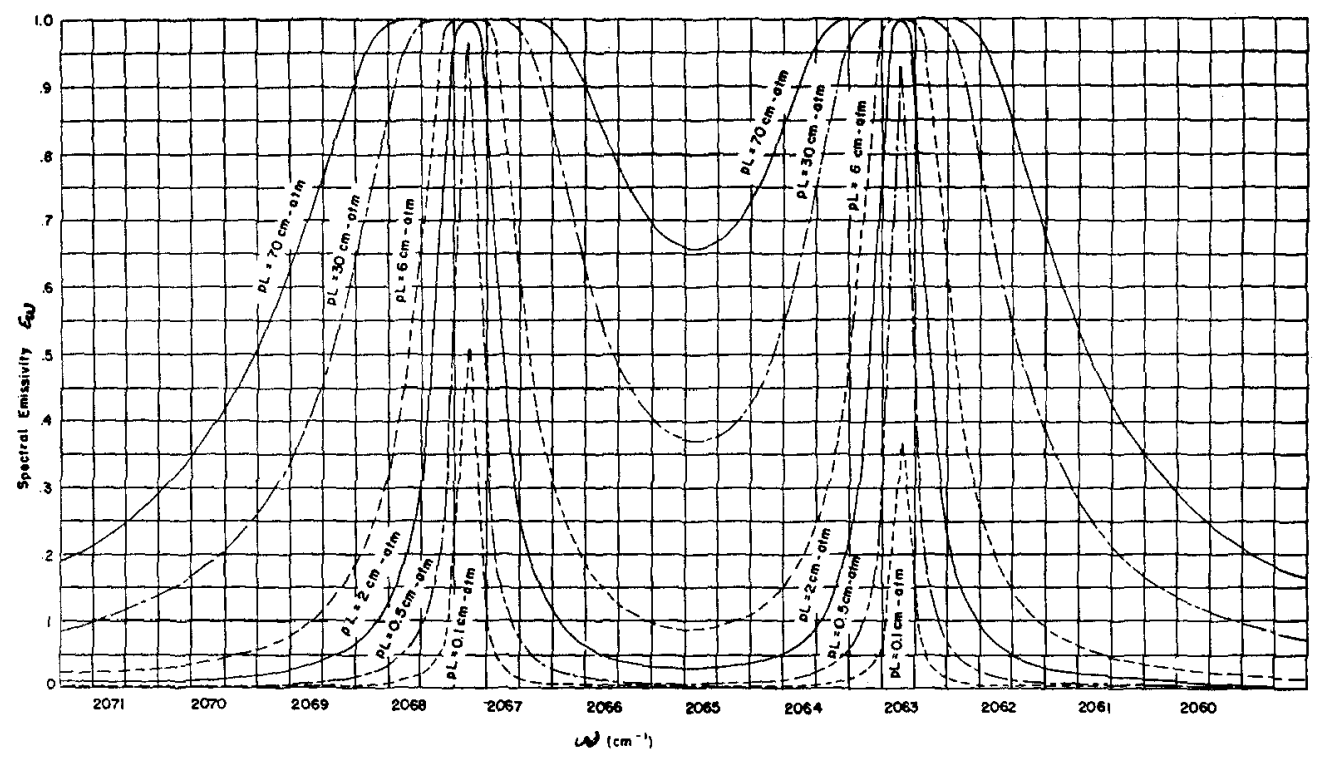

VA. Emissivity Calculations for $\mathrm{CO}$ at $300^{\circ} \mathrm{K}$ for Nonoverlapping Rotational Lines, Assuming Equal Spacing and Intensity of Lines

From Eqs. (3) and (4) it is apparent that

$$
E \simeq\left(\bar{R}^{0} / \sigma T^{4}\right) \int_{0}^{\infty}\left[1-\exp \left(-P_{\omega} X\right)\right] d \omega,
$$

where $\vec{R}^{0}$ represents an average value for $R^{0}(\omega)$ in the wave number interval for which $P_{\omega}$ is sensibly different from zero. The principal contribution to radiant heat transfer arises in the present problem from the fundamental vibration-rotation band. For moderate values of the optical density and nonoverlapping, equally spaced, and equally intense rotational lines it is well-known that ${ }^{4}$

$$
\int_{0}^{\infty}\left[1-\exp \left(-P_{\omega} X\right)\right] d \omega=\Delta \omega_{F} \cdot 2\left(\bar{S}_{F} b X\right)^{\frac{1}{2}} / q .
$$

Here $\Delta \omega_{F}$ is the effective bandwidth, ${ }^{6} \bar{S}_{F}$ represents the integrated absorption of the equally intense rotational lines ${ }^{4,13}$ for the fundamental vibration-rotation band of $\mathrm{CO}$, and $q$ is the spacing of the equally spaced rotational lines. Combining Eqs. (12) and (13) leads to the result

$$
E=2\left(\bar{R}^{0} \Delta \omega_{F} / \sigma T^{4}\right)\left(\bar{S}_{F} b X\right)^{\frac{1}{2}} / q .
$$

As is known from the known limits of validity of the assumption that the lines are equally spaced and equally intense, Eq. (14) predicts accurately all of the qualitative features of an exact numerical solution for small values of $X$. Thus

and

$$
\partial E / \partial(X)^{\frac{1}{2}}=\text { constant for fixed } b,
$$

$$
\partial E / \partial(b)^{\frac{1}{2}}=\text { constant for fixed } X .
$$

Actually, we can do better than predict the functional form of $E$. Thus it has been shown ${ }^{13}$ that for $\mathrm{CO}$ at $300^{\circ} \mathrm{K}$

$$
\bar{S}_{F}=1.9 \mathrm{~cm}^{-2} \text { atmos }^{-1} .
$$

Also $\Delta \omega_{F} \approx 250 \mathrm{~cm}^{-1}$ extending from 2000 to $2250 \mathrm{~cm}^{-1}$, $q=4 \mathrm{~cm}^{-1}$, and $b=0.08 \mathrm{~cm}^{-1}$. Hence, Eq. (12) becomes at $300^{\circ} \mathrm{K}$

$$
E \simeq 1.48 \times 10^{-3}(X)^{\frac{3}{3}}
$$

where $X$ is in cm-atmos, $b=0.08 \mathrm{~cm}$.

The emissivity $E$ calculated for $\mathrm{CO}$ at $300^{\circ} \mathrm{K}$ for a rotational half-width of $0.08 \mathrm{~cm}^{-1}$ (cf. Table III) is plotted as a function of $(X)^{\frac{1}{3}}$ in Fig. 4. Reference to Fig. 4 shows that

$$
\begin{aligned}
& E=1.64 \times 10^{-3}(X)^{\frac{1}{2}} \\
& \left(b=0.08 \mathrm{~cm}^{-1}, 0.1 \leq X \leq 2 \mathrm{~cm} \text {-atmos }\right) .
\end{aligned}
$$

Comparison of Eqs. (16a) and (16b) shows that we can predict absolute emissivity values with an accuracy of about 10 percent without performing numerical calculations.

Considering that the objective of the present investigations is the theoretical calculation of emissivities from spectroscopic data, the following objections may be raised to the use of Eq. (14): (1) the absolute error involved in the use of Eq. (14) cannot be estimated without performing accurate numerical calculations; (2) Eq. (15) is an empirical relation whence it follows that the dependence of $\bar{S}_{F}$ on temperature and total pressure cannot be predicted accurately. Nevertheless, particularly in view of the fact that Elsasser ${ }^{4}$ has obtained an integral representation for partial overlapping between equally spaced and equally intense rotational lines, the use of simplified treatments of the type given in Eq. (14) may be indicated in some cases. Thus we note that as the temperature is increased $\bar{P}_{F}$ must approach the ratio of integrated absorption to bandwidth, ${ }^{8}$ a quantity which can be calculated ${ }^{6}$ without difficulty. 


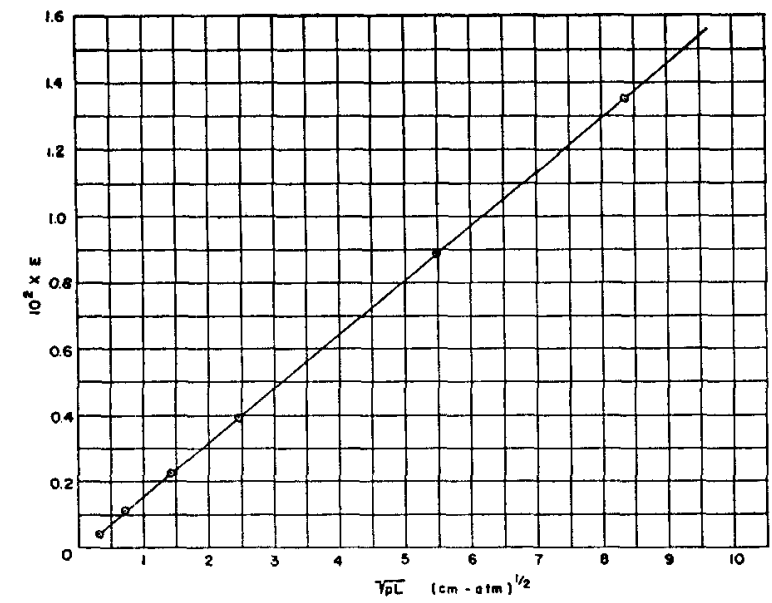

Fig. 4. Calculated emissivity $E$ as a function of the square root of the optical density at $300^{\circ} \mathrm{K}$ and atmospheric pressure $\left(b=0.08 \mathrm{~cm}^{-1}\right)$.

\section{VB. Emissivity Calculations for $\mathrm{CO}$ at $300^{\circ} \mathrm{K}$ for Nonoverlapping Rotational Lines Using Asymptotic Expressions for the Modified Bessel Functions}

The emission functions

$$
f(x)=x \exp (-x)\left[I_{0}(x)+I_{1}(x)\right]
$$

appearing in Eq. (8) have been studied and evaluated by Elsasser ${ }^{4}$ who has emphasized the fact that, even for moderate numerical values of $x$, asymptotic expressions for the modified Bessel functions yield very good approximations to the value of $f(x)$. However, this result has not heretofore been utilized in connection with accurate treatments on nonoverlapping rotational lines in which proper allowance is made for the change in intensity from one rotational line to another. It is the purpose of the present discussion to evaluate the integral appearing in Eq. (8) and to determine the value of the emissivity $E$ from Eq. (4) under conditions where the asymptotic expressions for the modified Bessel functions can be used.

Before describing the details of the integration it may be in order to emphasize the fact that the results of the present approximate treatment must be used with caution since the analysis rests on two seemingly contradictory assumptions. Thus overlapping between rotational lines is minimized by small values of $x$ (compare Section IV), whereas the asymptotic expressions for the modified Bessel functions apply most accurately for large values of $x$. Fortunately a range of values of $x$ exists (particularly at low pressures where the rotational halfwidth is small) for diatomic molecules with relatively distant rotational spacing, where both restrictive conditions are satisfied closely. In particular, for the problem under discussion, it has already been shown that overlapping between rotational lines is unimportant for optical densities smaller than about $2 \mathrm{~cm}$-atmos (com- pare Sections III and IV). Furthermore, the range of values of integrated absorption for the rotational lines of the fundamental vibration-rotation band (cf. Table I) is such that the error made by setting ${ }^{4}$

$$
f(x)=2(x / 2 \pi)^{\frac{1}{2}}
$$

is small for most of the rotational lines, provided the optical density is greater than about $0.25 \mathrm{~cm}$-atmos. $\|$ Hence, it is to be expected that a useful approximate solution will be obtained at least for $0.25 \mathrm{~cm}$-atmos $\leq p L \leq 2.0 \mathrm{~cm}$-atmos. The approximate treatment utilizing asymptotic expressions for the modified Bessel functions is, of course, not applicable to calculations on the first overtone because of the small values of $S_{j}{ }^{\prime}$, $S_{j-1}^{\prime}$ (cf. Table II). However, as was noted in Sections III and VA, the contribution to the emissivity $E$ from the first overtone is negligibly small in the present problem.

Utilizing the approximation expressed by Eq. (18) in Eq. (8) it is found from Eq. (4) that

$$
\begin{gathered}
E=30(u / \pi)^{4} \sum_{j=1}^{\infty}\left\{\frac{\left(\omega_{j} / \omega^{*}\right)^{3}}{\left[\exp \left(u \omega_{j} / \omega^{*}\right)\right]-1}\left[b S_{j} X /\left(\omega^{*}\right)^{2}\right]^{3}\right. \\
\left.+\frac{\left(\omega_{j} / \omega^{*}\right)^{3}}{\left[\exp \left(u \omega_{j-1} / \omega^{*}\right)\right]-1}\left[b S_{j-1} X /\left(\omega^{*}\right)^{2}\right]^{\frac{1}{2}}\right\},
\end{gathered}
$$

where explicit relations have been introduced for the Stefan-Boltzmann constant and, by using the wellknown Planck distribution law, for the spectral radiation intensity emitted from a blackbody. Here we have adopted the notation of Mayer and Mayer $^{20}$ with

$$
u=h c \omega^{*} / k T=h \nu^{*} / k T \text {. }
$$

In terms of the customary notation for the spectroscopic constants $^{21}$ we now introduce the following close approximations, neglecting terms involving $(\gamma j)^{3}$ and higher,

$$
\begin{gathered}
\omega_{j} / \omega^{*}=1-2 \gamma j-(\delta / \gamma) \gamma^{2} j^{2}, \\
F^{*}=1+4 \gamma j-3 \gamma^{2} j^{2},
\end{gathered}
$$

$$
\begin{aligned}
& G^{2}\left[\exp \left(u \omega_{j} / \omega^{*}\right)-1\right]^{-1} \\
& =e^{-u}\left(1-e^{-u}\right)^{-1}\left\{1+\gamma j u\left[2+e^{-u}\left(1-e^{-u}\right)^{-1}\right]\right. \\
& +\gamma^{2} j^{2}\left[u(2 u+\delta / \gamma)+u e^{-u}\left(1-e^{-u}\right)^{-1}(3 u+\delta / 2 \gamma)\right. \\
& \left.\left.+(3 / 2) u^{2} e^{-2 u}\left(1-e^{-u}\right)^{-2}\right]\right\},
\end{aligned}
$$

$\|$ By utilizing the known values of $f(x)$ and of $S_{j}, S_{j-1}$ it is readily shown that the error involved in using asymptotic forms for the Bessel functions is

less than 5 percent for $1 \leq j \leq 18$ for $X=2 \mathrm{~cm}$-atmos,

less than 5 percent for $2 \leq j \leq 16$ for $X=1 \mathrm{~cm}$-atmos,

less than 15 percent for $2 \leq j \leq 14$ for $X=0.25 \mathrm{~cm}$-atmos, etc.

Since most of the contributions to $E$ result from the rotational lines with $1 \leq j \leq 16$, the preceding statement suggests the range of validity of the present treatment.

${ }^{20} \mathrm{~J}$. E. Mayer and M. G. Mayer, Statistical Mechanics (John Wiley and Sons, Inc., New York, 1940).

${ }^{21}$ A compilation of numerical values may be found on p. 468 of reference 20 . 
$\exp \{-[E(0, j)-E(0,0)] / 2 k T\}$

$$
=\left[\exp \left(-\gamma j^{2} u / 2\right)\right]\left[1-(\gamma j u / 2)+\left(\gamma^{2} j^{2} u^{2} / 8\right)\right],
$$

and ${ }^{20}$

$Q \exp [E(0,0) / k T]$

$$
\begin{aligned}
& =(\gamma u)^{-1}\left(1-e^{-u}\right)^{-1}[1+\gamma(u / 3+8 / u) \\
& \left.+\delta\left(e^{u}-1\right)^{-1}+2 x^{*} u\left(e^{u}-1\right)^{-2}\right] .
\end{aligned}
$$

The relations corresponding to Eqs. (20) to (23) for the transitions $j-1 \rightarrow j$ are obtained by replacing $j$ by $-j$. Because of this symmetry, the sum of terms from the transitions $j \rightarrow j-1$ and $j-1 \rightarrow j$ for every $j$ is a function of $j^{2}$ only, and the linear terms in $j$ will cancel. Hence the following result is obtained from Eq. (19), Eqs. (2a), (20) to (23), and Eq. (2b) together with the relations corresponding to Eqs. (20) to (23) for the transitions $j-1 \rightarrow j$ :

$$
\begin{gathered}
E=30 u^{5} \pi^{-4} e^{-u} f\left(\gamma, \delta, x^{*}, u\right)\left[\left(\gamma b / \omega^{*}\right)\left(A X / \omega^{*}\right)\right]^{\frac{k}{*}} . \\
\sum_{v^{\prime}} 2 j^{\frac{1}{2}} e^{-\gamma u^{2} / 2}\left[1+\gamma^{2} j^{2} g(\gamma, \delta, u)\right],
\end{gathered}
$$

where the temperature-independent quantity $A$ is given by the relation

$$
A=\left(N_{T} \pi \epsilon^{2} / 3 \mu c^{2} u\right)\left(\omega^{*} / \omega_{e}\right) .
$$

The function $f\left(\gamma, \delta, x^{*}, u\right)$, which is obtained from Eq. (24),

$$
\begin{aligned}
f\left(\gamma, \delta, x^{*}, u\right)=1- & \gamma(u / 6+4 / u) \\
- & \delta\left[2\left(e^{u}-1\right)\right]^{-1}-x^{*} u\left(e^{u}-1\right)^{-2},
\end{aligned}
$$

is evidently close to unity. The function $g(\gamma, \delta, u)$ is

$$
\begin{aligned}
g(\gamma, \delta, u)= & (3 / 2)\left(u e^{-u}\right)^{2}\left(1-e^{-u}\right)^{-2} \\
& +u e^{-u}\left(1-e^{-u}\right)^{-1}(5 u / 2+\delta / 2 \gamma-3) \\
& +u(\delta / \gamma-9 / 2+9 u / 8)+27 / 2-7 \delta / 2 \gamma .
\end{aligned}
$$

The Euler-Maclaurin summation formula ${ }^{22}$ can now be employed to evaluate the summation over $j$ appearing in Eq. (25). Details are given in the Appendix. The result is

$$
\begin{aligned}
E=30 u^{5} \pi^{-4} e^{-u} & {\left[\left(\gamma b / \omega^{*}\right)\left(A X / \omega^{*}\right)\right]^{\frac{1}{2}} } \\
& \times f\left(\gamma, \delta, x^{*}, u\right)\left\{1.225(2 / \gamma u)^{z}\right. \\
& \times[1+(3 \gamma / 2 u) g(\gamma, \delta, u)]-0.417\} .
\end{aligned}
$$

Since $f\left(\gamma, \delta, x^{*}, u\right)$ is close to unity and, at room temperature,

$$
(3 \gamma / 2 u) g(\gamma, \delta, u) \ll 1, \quad 0.417 \ll 1.255(2 / \gamma u)^{\frac{3}{2}},
$$

the following close approximation is obtained:

$$
E=0.6345 u^{5} e^{-u}(\gamma u)^{-*}\left[\left(\gamma b / \omega^{*}\right)\left(A X / \omega^{*}\right)\right]^{\frac{1}{2}} .
$$

Utilizing the spectroscopic constants of Sponer ${ }^{14}$ for

\footnotetext{
${ }^{22}$ See, for example, reference 20, p. 431 .
}

$\mathrm{CO}$ and setting ${ }^{12}$

$$
A=22.95 \mathrm{~cm}^{-2} \text { atmos }^{-1} \text {, }
$$

it is found from Eq. (29) for $b=0.08 \mathrm{~cm}^{-1}$ that

$E=1.67 \times 10^{-3}(X)^{\frac{1}{2}}$

( $X$ in cm-atmos, $b=0.08 \mathrm{~cm}^{-1}$ ).

(16c)

The expression for $E$ given in Eq. (16c) is seen to be in excellent agreement with the result of numerical calculations given in Eq. (16b).$^{23}$ Hence, we conclude that Eqs. (29) and (30) represent useful approximations for nonoverlapping rotational lines under appropriate conditions. It is evident from the previous discussion that Eq. (16c) cannot apply, even approximately, for values of $X$ much smaller than $0.25 \mathrm{~cm}$-atmos. This conclusion is in agreement with the well-known fact that the absorption of radiation is not proportional to the square root of the optical density for very small values of the optical density.

The expression given for $E$ in Eq. (29) represents a significant improvement over Eq. (14) (which was derived from Elsasser's treatment for equally spaced and equally intense rotational lines). Thus the parameters appearing in Eq. (29) are, in most cases, well-known molecular constants. However, Eq. (29) must be used with discretion, particularly as regards the calculation of emissivity as a function of temperature. ${ }^{24}$

\section{APPENDIX}

Application of the Euler-Maclaurin summation formula $^{22}$ to the sum appearing in Eq. (25) leads to the relation

$$
\begin{aligned}
2 \sum_{j=1}^{\infty} j^{\frac{1}{2}} e^{-\gamma u j^{2} / 2}\left[1+\gamma^{2} j^{2} g(\gamma, \delta, u)\right] \simeq 2 \int_{1}^{\infty} j^{\frac{1}{2}} e^{-\gamma u j^{2} / 2} \\
\times\left[1+\gamma^{2} j^{2} g(\gamma, \delta, u)\right] d j+(11 / 12) .
\end{aligned}
$$

The integral from 1 to $\infty$ can be expressed as the difference between the integral from 0 to $\infty$ and from 0 to 1 . The integral from 0 to 1 can be evaluated approximately as $4 / 3$. Therefore,

$$
\begin{aligned}
& 2 \sum_{j=1}^{\infty} j^{\frac{1}{2}} e^{-\gamma u j^{2} / 2}\left[1+\gamma^{2} j^{2} g(\gamma, \delta, u)\right] \\
& \quad \simeq 2 \int_{0}^{\infty} j^{\frac{1}{2}} e^{-\gamma u j^{2} / 2}\left[1+\gamma^{2} j^{2} g(\gamma, \delta, u)\right] d j-5 / 12 \\
& \quad=\Gamma(3 / 4)(2 / \gamma u)^{\frac{3}{3}}[1+(3 \gamma / 2 u) g(\gamma, \delta, u)]-5 / 12 \text {. (A2). }
\end{aligned}
$$

${ }^{23}$ The fact that the present analysis leads to emissivities which are somewhat too large is a necessary consequence of the use of the asymptotic relation given in Eq. (18), which overestimates $f(x)$ for every finite value of $x$.

${ }^{24}$ Compare Section IV of reference 9. 